\title{
Metabolic control after kidney and pancreas transplantation: whole series results and effects of segmental duct obstruction versus whole pancreas with bladder diversion technique
}

\author{
R. Caldara ${ }^{1}$, X. Martin ${ }^{2}$, A. Secchi ${ }^{1}$, N. Lefrancois ${ }^{2}$, J. L. Touraine ${ }^{2}$, G. Pozza ${ }^{1}$ and J. M. Dubernard ${ }^{2}$ \\ ${ }^{1}$ Istituto Scientifico San Raffaele, Milan, Italy and ${ }^{2}$ Hôpital E. Herriot, Lyon, France
}

Summary. From October 1976 to December 1990 181 pancreatic transplants were performed in our centre on 171 Type 1 ( insulin-dependent) diabetic patients. Oral glucose tolerance test evaluated 1 year after surgery in 31 subjects showed an impaired glucose tolerance at $120 \mathrm{~min}$ (blood glucose $9.5 \pm 0.6 \mathrm{mmol} / \mathrm{l}$ ). Similar results were obtained in seven patients 3 years after transplantation (blood glucose at $120 \mathrm{~min}$ $8.3 \pm 1.08 \mathrm{mmol} / \mathrm{l})$. $24 \mathrm{~h}$ metabolic profiles performed at the same intervals showed near normal blood glucose levels and good insulin release. Preliminary data concerning a randomized, comparative study between whole pancreas with bladder diversion and segmental pancreas transplantation, showed better metabolic control in the patients who received the whole pancreas, probably due to the greater islet mass grafted.

Key words: Pancreas transplantation - Bladder diversion - Oral Glucose Tolerance Test - Type 1 (insulin-dependent) diabetes mellitus

\section{Introduction}

Pancreas transplantation is performed in Type 1 (insulin-dependent) diabetic patients in order to restore a self-regulated source of insulin (Lillehei 1970; Pozza 1983).

From October 1976 to December 1990, 181 pancreatic transplants were performed in our center on 171 Type 1 diabetic patients.

We report metabolic data of patients with a functioning pancreatic graft longer than 1 and 3 years (Study 1).

To evaluate the impact of the transplanted pancreatic mass, from December 1988 to December 1990 we perfomed a randomized, comparative study between 26 segmental pancreas with duct obstruction by neoprene and 25 pancreatico-duodenal transplantation with bladder diversion. We report the preliminary metabolic data observed 6 months after surgery (Study 2).

\section{Subjects and methods}

Study1. From October 1976 to December 1990 we performed 181 pancreas transplantations in 171 Type 1 (insulin-dependent) diabetic patients. The mean age of patients was 37.0 0.1 years and duration of diabetes was $22.6 \pm 0.6$ years, $86 \%$ of patients were on dialysis with dialysis duration of $13 \pm 3$ months. Simultaneous kidney transplantation was performed on 157 patients while in 24 cases the patients underwent pancreas transplantation alone.

In 129 cases a segmental, neoprene injected pancreas graft was used. Fourteen patients received a pancreatico-duodenal graft with enteric diversion and 25 patients underwent a pancreatico-duodenal transplantation with bladder diversion. Two patients received a whole, neoprene injected pancreas. We employed different immunosuppressive treatments. The last protocol, used from March 1990 , consisted of a quadruple drug regimen of azathioprine (AZA) 23 mg.kg- ${ }^{1} \cdot$ day $^{-1}$ then 1 mg.kg-1.day ${ }^{-1}$, Cyclosporin A (CYA) 2 mg. $\mathrm{kg}^{-1}$.day ${ }^{-1}$ i.v. then $6 \mathrm{mg}^{-\mathrm{kg}^{-1}}$.day $^{-1}$ orally, Prednisone $1 \mathrm{mg} . \mathrm{kg}^{-}$ ${ }^{1}$.day ${ }^{-1}$ then tapered to $0.25 \mathrm{mg} \cdot \mathrm{kg}^{-1}$.day ${ }^{-1}$ and anti-thymocyteglobulins (ATG) or anti-T-cell monoclonal antibodies (OKT3) during the first 2 weeks. All patients underwent a $24 \mathrm{~h}$ metabolic profile (blood samples for blood glucose and serum free insulin at 08.00 hours, 10.00 hours, 12.00 hours, 02.00 hours pm, 06.00 hours pm, 09.00 hours pm, 12.00 hours pm) and an oral glucose tolerance test (OGTT, glucose $75 \mathrm{~g}$ p.o. : blood samples for blood glucose and serum free insulin at $0,30,60,90,120$ and $180 \mathrm{~min}$ ) every year after surgery. Glycosylated heamoglobin (HbAlc) was evaluated at the same intervals.

Study2. From December 1988 to December 1990 we performed a randomized, comparative study between 25 whole pancreas with bladder diversion (group BD) and 26 , duct obstructed by neoprene, segmental pancreas (group DO). The mean age of patients and Type 1 diabetes duration was $36.9 \pm 2.6$ years and $23.3 \pm 1 . .6$ years in group $\mathrm{BD}$ and $40 \pm 1.6$ years and $24.8 \pm 6.5$ years in group DO. For each group 19 patients were on dialysis with a duration of $17.8 \pm 5$ months in group $B D$ and $9 \pm 3$ in group $D O$, respectively. For technical reasons in two patients of group BD a conversion of their whole pancreas to a segmental pancreas transplantation was necessary. 
Immunosuppressive treatment was based on a quadruple regimen ( ATG or OKT3, AZA, CYA, prednisone) in both groups. Patients who received a pancreas graft before March 1990 received lower doses of Prednisone (15 mg on the first day then $0.25 \mathrm{mg} \cdot \mathrm{kg}^{-1} \cdot \mathrm{day}^{-1}$ vs $1 \mathrm{mg} \cdot \mathrm{kg}^{-1} \cdot$ day $^{-1}$ during the post-operative period, tapered to $0.25 \mathrm{mg} \cdot \mathrm{kg}^{-1}$.day 1) All patients underwent an OGTT 6 months after surgery.

Statistical analysis was based on Mann-Whitney test.

\section{Results}

Study 1

Complete insulin-independence was achieved in all cases of functioning graft. One year after operation 31 patients were submitted to an OGTT : fasting blood glucose levels were in the normal range (4.9 \pm 0.1 $\mathrm{mmo} / 1$ ), but an impaired glucose tolerance, according to WHO criteria, was observed at $120 \mathrm{~min} \quad(9.5 \pm 0.6$ mmol/1). Similar results were obtained in seven patients studied 3 years after transplantation (fasting blood glucose $4.7 \pm 0.2 \mathrm{mmol} / \mathrm{l}$, at $120 \mathrm{~min} 8.3 \pm 1.08$ $\mathrm{mmol} / \mathrm{l})$. A $24 \mathrm{~h}$ metabolic profile performed in 28 patients 1 year after surgery showed normal blood glucose levels and good insulin release. The values recorded after 3 years in 5 patients confirmed a good metabolic control (blood glucose $\mathrm{mmol} / \mathrm{l}$ at 08.00 hours $4.9 \pm 0.2$, at 02.00 hours p.m. $5.4 \pm 0.3$, at 06.00 hours p.m. 6.1 \pm 0.4 ; serum free insulin $\mu \mathrm{U} / \mathrm{ml}$ at 08.00 hours $13.3 \pm 4.9$, at 02.00 hours p.m $31.6 \pm 11.6$, at 06.00 hours p.m $36.7 \pm 14.5$ ). HbAlc values were in the normal range during the all follow-up period (HbA1c 3 years after transplantation 5.3 $\pm 0.2 \%$ )

\section{Study 2}

The comparative study, performed in four patients of group BD and in four patients of group DO, 6 months after surgery, showed similar fasting blood glucose levels (group $\mathrm{BD}$ vs group $\mathrm{DO} 5.0 \pm 0.2$ vs $5.4 \pm 0.1$ mmol/1). After an OGTT an impaired glucose tolerance was observed in group DO (blood glucose $\mathrm{mmol} / \mathrm{l}$ at $120 \mathrm{~min} 7.4 \pm 1.5$ ) while patients of the BD group showed a normal glucose tolerance (blood glucose at $120 \min 5.8 \pm 0.5$ ) (Fig.1).

Both groups showed a good insulin release after the same test (basal serum free insulin $\mu \mathrm{U} / \mathrm{ml}$ group $\mathrm{BD}$ vs DO $14.6 \pm 4.8$ vs $16.1 \pm 8.1$; at $120 \min 43.0 \pm 13.1$ vs $34.6 \pm 15.7$ ) (Fig.2). The difference between the two groups was not statistically significant.

\section{Discussion}

All patients with functioning graft achieved a satisfactory metabolic control within a few weeks. The results of the metabolic investigations showed a near normal short- and long-term endocrine function. The findings of impaired glucose tolerance during OGTT could be the consequence of several factors such as: steroid administration, the non-portal but systemic insulin drainage, defect of extra-pancreatic factors,

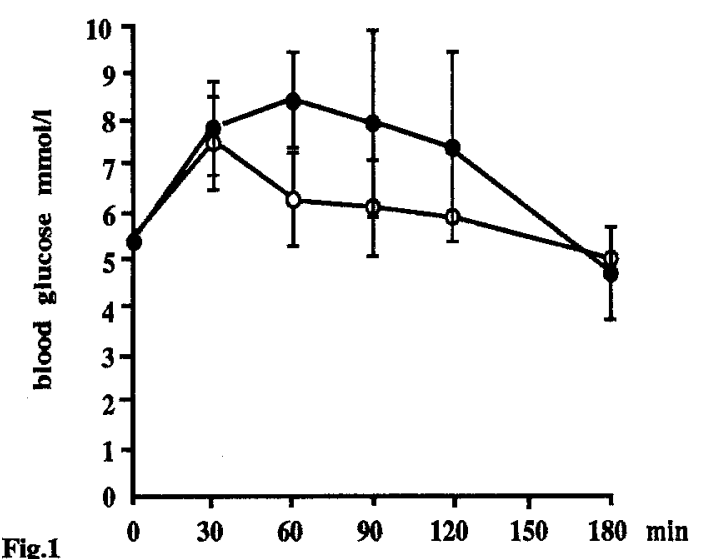

Oral glucose tolerance test 6 months after transplantation: blood glucose levels (mmol/l)

O $\mathrm{BD}$ (4 patients) $\mathrm{DO}$ (4 patients)

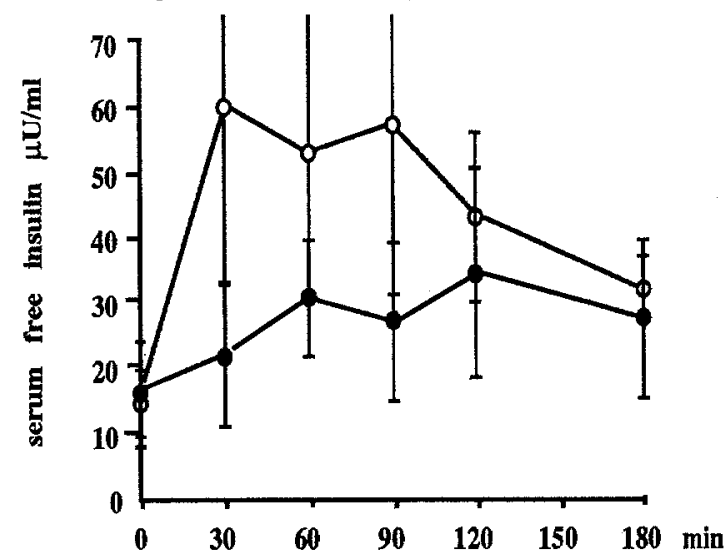

Fig. 2

Oral glucose tolerance test 6 months after transplantation: serum free insulin $(\mu \mathrm{U} / \mathrm{ml})$

O BD (4 patients) DO (4 patients)

rejection. Preliminary data of our randomized, comparative trial confirm data previously shown with the digestive technique (Secchi 1991). A better metabolic control, probably the consequence of the greater islet mass grafted, in the patients who received the entire pancreas was observed.

\section{References}

Lillehei R.C., Simmons R.L., Najarian J.S. et al (1970) Pancreatico duodenal allotransplantation. Experimental and clinical experience. Ann. Surg 172:405-436

Pozza G., Traeger J., Dubernard J.M., Secchi A. et al (1983)

Endocrine responses of Type I (insulin- dependent) diabetic patients following succesful pancreas transplantation. Diabetologia 24:244-248

Secchi A., Dubernard J.M., La Rocca E., Lefrancois N., Melandri M., Martin X., Touraine J.L., Traeger J., Pozza G. (1991) Endocrinometabolic effects of whole versus segmental pancreas allotransplantation in diabetic patients, a two years follow-up.

Transplantation $51: 625-629$

Dr. R. Caldara

Istituto Scientifico San Raffaele

Department of Internal Medicine

via Olgettina 60

20132 Milan - Italy 